\title{
SUBELLIPTIC CORDES ESTIMATES IN THE GRUŠIN PLANE
}

\author{
G. DI FAZIO, A. DOMOKOS, M. S. FANCIULLO, AND J. J. MANFREDI
}

\begin{abstract}
We apply subelliptic Cordes conditions and Talenti-Pucci type inequalities to prove $W^{2,2}$ and $C^{1, \alpha}$ regularities for $p$-harmonic functions in the Grušin plane.
\end{abstract}

\section{INTRODUCTION}

The purpose of this paper is to prove interior regularity results for subelliptic p-harmonic functions in the case of the so-called Grušin vector fields $X_{1}=\frac{\partial}{\partial x}$ and $X_{2}=x \frac{\partial}{\partial t}$. Subelliptic p-harmonic functions are weak solutions of the corresponding p-Laplace equation

$$
-\Delta_{X}^{p} u=-X_{1}\left(|X u|^{p-2} X_{1} u\right)-X_{2}\left(|X u|^{p-2} X_{2} u\right)=0, \text { in } \Omega .
$$

Our goal is to obtain new Talenti-Pucci type inequalities (see Remark 3.2 and Theorem 3.4) and use them to obtain regularity for the weak solution of the quasilinear equation (1). For example, we show that the following sharp inequality holds:

(2) $\iint_{\mathbb{R}^{2}}\left(\frac{\partial u}{\partial t}\right)^{2} d x d t \leq \iint_{\mathbb{R}^{2}}\left(\frac{\partial^{2} u}{\partial x^{2}}+x^{2} \frac{\partial^{2} u}{\partial t^{2}}\right)^{2} d x d t$, for all $u \in C_{0}^{\infty}\left(\mathbb{R}^{2}\right)$.

When $p=2$ the corresponding subelliptic Laplacian $\Delta_{X}$ is hypoelliptic; in particular $u$ is a $C^{\infty}$ function on any open subset of $\Omega$ where $\Delta_{X} u$ is. In 14] Hörmander proved this regularity result in the case of an arbitrary number of vector fields satisfying the bracket generating (or Hörmander type) condition. Another approach, valid in $\mathbb{R}^{n}$ for a system of Hörmander type vector fields, can be found in the paper of Rothschild and Stein ([20]) where they apply a lifting process to the vector fields to get a new system of free vector fields. This new system can be approximated by a system of left invariant vector fields from the Lie algebra of a nilpotent Lie group. It is then possible to use the results of Folland [9, 10] to get a parametrix for the lifted subelliptic Laplacian, which inverts it modulo a smoothing operator. Then a projection reverts the lifting process to obtain a parametrix for the initial Laplacian.

Unfortunately, this process does not seem to work for the nonlinear case. Thus, we are forced to try a direct approach to the regularity problem obtained by the lifting method.

We briefly describe the content of the paper. In the second section we introduce the lifting process and the approximation theorem by Rothschild

Date: July 28, 2007.

Key words and phrases. Carnot-Carthéodory metric, Grušin plane, p-Laplacian.

Fourth author partially supported by NSF award DMS-0500983. 
and Stein about a system of Hörmander vector fields. In the third section we prove the Talenti-Pucci type inequality for functions from the space $W_{0}^{2, p}(\Omega)$. In the fourth section we get a regularity result for solutions of a linear equation in non-divergence form with measurable coefficients satisfying the Cordes condition. In the fifth and sixth sections we obtain $W^{2,2}$ and $C^{1, \alpha}$ for the subelliptic $p$-Laplacian.

\section{Carnot-Carathéodory Metric Spaces in $\mathbb{R}^{n}$}

Let us consider a system $X=\left\{X_{1}, X_{2}, \cdots, X_{q}\right\}$ of $C^{\infty}$ vector fields defined in $\mathbb{R}^{n}, q \leq n$.

Definition 2.1 (Hörmander condition). We say that the system $X$ satisfies the Hörmander condition of step $m$ at the point $x_{0} \in \mathbb{R}^{n}$, if the vector fields $X_{i}$ together with their commutators of length at most $m$ span $\mathbb{R}^{n}$ at $x_{0}$.

Given the system $X$ of vector fields we define a distance, which is usually called the Carnot-Carathéodory distance, in $\mathbb{R}^{n}$ as follows.

An absolute continuous curve $\gamma:[0, T] \rightarrow \mathbb{R}^{n}$ is said to be $X$-subunitary if there exists a measurable vector function $h=\left(h_{1}, \ldots, h_{q}\right):[0, T] \rightarrow \mathbb{R}^{q}$ such that $\dot{\gamma}(t)=\sum_{i=1}^{q} h_{i}(t) X_{i}(\gamma(t))$ for a.e. $t \in[0, T]$ and $\|h\|_{\infty} \leq 1$. It is well known (see [4]) that, if the vector fields satisfy the Hörmander condition in every point of $\mathbb{R}^{n}$, the set of $X$-subunitary curves connecting two given points $x, y$ in $\mathbb{R}^{n}$ is not empty.

Definition 2.2. For $x, y \in \mathbb{R}^{n}$ we set the Carnot-Carathéodory distance between $x$ and $y$ as

$$
\begin{array}{r}
d(x, y)=\inf \left\{T \geq 0: \exists \text { an } X \text {-subunitary curve } \gamma:[0, T] \rightarrow \mathbb{R}^{n},\right. \\
\text { such that } \gamma(0)=x \text { and } \gamma(T)=y\} .
\end{array}
$$

For $x \in \mathbb{R}^{n}$ and $R>0$, we denote by

$$
B_{R}(x)=B(x, R)=\left\{y \in R^{n}: d(x, y)<R\right\}
$$

the Carnot-Carathéodory metric ball centered at $x$ with radius $R$. Next we set $\left|B_{R}(x)\right|$ to be the Lebesgue measure of the ball.

Denote by $Y_{1}, \ldots, Y_{l}$ the collection of $X_{j}$ and of those commutators which are needed to span $\mathbb{R}^{n}$. For any $i=1, \ldots, l$ we denote by $\operatorname{deg}\left(Y_{i}\right)$ the order of the commutator $Y_{i}$. If $I=\left(i_{1}, \ldots, i_{n}\right), 1 \leq i_{j} \leq l$, is a $n$-tuple of integers we call $d(I)=\sum_{j=1}^{n} \operatorname{deg}\left(Y_{i_{j}}\right)$ and $a_{I}=\operatorname{det}\left(Y_{i_{1}}, \ldots, Y_{i_{n}}\right)$. For a bounded $E \subset \mathbb{R}^{n}$ we set $Q=\sup \left\{d(I):\left|a_{I}(x)\right| \neq 0, x \in E\right\}$. The number $Q$ is called the local homogeneous dimension of $E$. The following result is a consequence of a theorem due to Nagel, Stein and Wainger (see [18]).

Theorem 2.1. For any bounded $E \subset \Omega$ there exists a constant $R_{0}$, depending on the system $X$ of vector fields and $E$, such that for all $x \in E$ and $R<R_{0}$ it results

$$
\left|B_{2 R}(x)\right| \leq 2^{Q}\left|B_{R}(x)\right|
$$

By the Rothschild-Stein Lifting Theorem (stated below) the vector fields $X_{1}, X_{2}, \cdots, X_{q}$ satisfying the Hörmander condition, can be lifted to some 
new free vector fields $\tilde{X}_{1}, \tilde{X}_{2}, \cdots \tilde{X}_{q}$ on $\mathbb{R}^{N}, N>n$, by adding extra variables. This means that, regarding the new set of vector fields, the only relations between the commutators of order less than or equal to $m$ are the antisymmetric relation and the Jacobi identity.

Definition 2.3. We say that the vector fields $X_{1}, X_{2}, \cdots, X_{q}$ in $\mathbb{R}^{n}$, which satisfy the Hörmander condition of step $m$ at some point $x_{0}$, are free up to order $m$ at $x_{0}$ if $n$ is the dimension of the free Lie algebra $\mathfrak{g}(q, m)$ of step $m$ on $q$ generators.

Theorem 2.2 (Rothschild-Stein). Let $X_{1}, X_{2}, \cdots, X_{q}$ be $C^{\infty}$ vector fields satisfying the Hörmander condition of step $m$ at some point $x_{0} \in \mathbb{R}^{n}$. Then in terms of new variables $t_{n+1}, t_{n+2}, \cdots, t_{N}$, there exist smooth functions $\lambda_{i l}(x, t)$ defined in a neighborhood $\tilde{U}$ of $\xi_{0}=\left(x_{0}, 0\right) \in \mathbb{R}^{N}$ such that the vector fields $\tilde{X}_{i}$ given by

$$
\tilde{X}_{i}=X_{i}+\sum_{l=n+1}^{N} \lambda_{i l}(x, t) \frac{\partial}{\partial t_{l}}, \quad i=1,2, \cdots, q,
$$

satisfy Hörmander condition of step $m$ and are free up to step $m$ at every point in $\tilde{U}$.

Next we define a local system of coordinates around a point $\xi \in \mathbb{R}^{N}$. Let $\left\{\tilde{X}_{\alpha}(\xi)\right\}_{\alpha \in A}\left(A\right.$ is a set of multi-indices $\alpha$ ) be a basis of $\mathbb{R}^{N}$ for every $\xi \in \tilde{U}$. We denote by $\exp \left(\sum_{\alpha} u_{\alpha} \tilde{X}_{\alpha}\right)(\xi)$ the solution of

$$
\left\{\begin{array}{l}
\dot{\gamma}=\sum_{\alpha} u_{\alpha} \tilde{X}_{\alpha}(\gamma) \\
\gamma(0)=\xi
\end{array}\right.
$$

at time $t=1$. Then we call $\left(u_{\alpha}\right)_{\alpha \in A}$ a system of coordinates around $\xi$ and define $\theta_{\xi}(\eta)=\left(u_{\alpha}\right)_{\alpha \in A}$, where $\xi, \eta \in \tilde{U}$ and

$$
\eta=\exp \left(\sum_{\alpha} u_{\alpha} \tilde{X}_{\alpha}\right) \xi
$$

The following theorem is also from [20] (see Theorem 5, §7.)

Theorem 2.3 (Rothschild-Stein). Let $\tilde{X}_{1}, \cdots, \tilde{X}_{q}$ be free up to step $m$ in $\xi_{0}$. Then there exist neighborhoods $\tilde{V}$ and $\tilde{W}$ of $\xi_{0}$ in $\mathbb{R}^{N}$ and $U$ of 0 , with $\tilde{W} \subset \subset \tilde{V}$ such that

(a) $\theta_{\xi} \mid \tilde{V}$ is a diffeomorphism onto the image, for every $\xi \in \tilde{V}$,

(b) $U \subset \theta_{\xi}(\tilde{V})$, for every $\xi \in \tilde{W}$,

(c) $\theta: \tilde{V} \times \tilde{V} \rightarrow \mathbb{R}^{N}$, defined by $\theta(\xi, \eta)=\theta_{\xi}(\eta)$, is in $C^{\infty}(\tilde{V} \times \tilde{V})$, and

(d) in the coordinates given by $\theta_{\xi}$ we can write $\tilde{X}_{i}=Y_{i}+R_{i}^{\xi}$, where $Y_{i}$ are left invariant vector fields and $R_{i}^{\xi}$ are vector fields depending smoothly on $\xi$.

Let us consider the Lie algebra $\mathfrak{g}(q, m)$. Then, through the exponential mapping we relate it to the corresponding simply connected Lie group $\mathcal{G}(q, m)$. A natural group law is then defined by using the Campbell - Hausdorff formula. Namely

$$
\left(\sum_{\alpha \in A} u_{\alpha} \tilde{X}_{\alpha}\right) \circ\left(\sum_{\alpha \in A} v_{\alpha} \tilde{X}_{\alpha}\right)=\sum_{\alpha \in A}\left(u_{\alpha}+v_{\alpha}\right) \tilde{X}_{\alpha}+\frac{1}{2}\left[\sum_{\alpha \in A} u_{\alpha} \tilde{x}_{\alpha}, \sum_{\alpha \in A} v_{\alpha} \tilde{x}_{\alpha}\right]+\cdots
$$


In this group we can naturally define the dilations by

$$
\delta_{t}\left(\left(u_{\alpha}\right)_{\alpha \in A}\right)=\left(t^{|\alpha|} u_{\alpha}\right)_{\alpha \in A} .
$$

These dilations are automorphisms of the group $\mathcal{G}(q, m)$.

A function $k$ defined on $\mathcal{G}(q, m) \backslash\{0\}$, smooth away from the origin, is said to be of type $\lambda>0$, if $k\left(\delta_{t}(\xi)\right)=t^{\lambda-Q} k(\xi)$, for all $t>0$. $k$ is said to be of type 0 if the last equality holds with $\lambda=0$ and the mean value of $k$ vanishes, that is

$$
\int_{a \leq|u| \leq b} k(u) d u=0 \text { for all } a<b,
$$

where $|\cdot|$ is a suitable homogeneous norm on the group $\mathcal{G}(q, m)$ (see (5.2) in [20]).

Definition 2.4. A measurable function $K$ is called to be a kernel of type $\lambda \geq 0$ if for any positive integer $l$ we can write

$$
K(\xi, \eta)=\sum_{i=1}^{s} a_{i}(\xi) k_{\xi}^{(i)}(\theta(\eta, \xi)) b_{i}(\eta)+E_{l}(\xi, \eta),
$$

where $E_{l} \in C_{0}^{l}, a_{i}, b_{i} \in C_{0}^{\infty}$ and the functions $k_{\xi}^{(i)}$ are of type $\geq \lambda$ and depend smoothly on $\xi$.

Definition 2.5. An operator $T$ is said to be of type $\lambda>0$, if

$$
(T f)(\xi)=\int K(\xi, \eta) f(\eta) d \eta
$$

where $K$ is a kernel of type $\lambda$. An operator $T$ is said to be of type 0 if

$$
(T f)(\xi)=\int K(\xi, \eta) f(\eta) d \eta+a(\xi) f(\xi)
$$

where $K$ is a kernel of type 0 and $a \in C_{0}^{\infty}$.

We refer the reader to [20] for a thorough explanation.

\section{Second Derivative Estimates for sub-Laplacians}

Let $\Omega$ be a bounded subset of $\mathbb{R}^{n}$ and $X=\left(X_{1}, \ldots, X_{q}\right)$ be a system of Hörmander vector fields in $\Omega$. We set, with $k \in \mathbb{N}$,

$$
W^{k, p}(\Omega)=\left\{u: \Omega \rightarrow \mathbb{R}: u, X_{i_{1}} \ldots X_{i_{j}} u \in L^{p}(\Omega), 1 \leq j \leq k\right\},
$$

endowed with the norm

$$
\begin{gathered}
\|u\|_{W^{p, k}}=\|u\|_{L^{p}}+\sum_{h=1}^{k} \sum_{i_{j}=1}^{q}\left\|X_{i_{1}} X_{i_{2}} \ldots X_{i_{h}}\right\|_{L^{p}}, \\
W_{\text {loc }}^{k, p}(\Omega)=\left\{u: \Omega \rightarrow \mathbb{R}: \eta u \in W^{k, p}(\Omega), \text { for all } \eta \in C_{0}^{\infty}(\Omega)\right\},
\end{gathered}
$$

and $W_{0}^{k, p}(\Omega)$ as the closure of $C_{0}^{\infty}(\Omega)$ in $W^{k, p}(\Omega)$.

We denote by $\Delta_{X}$ the sub-elliptic Laplacian operator $\sum_{j=1}^{q} X_{j}^{2}$ and by $\Delta_{\tilde{X}}$ the operator $\sum_{j=1}^{q} \tilde{X}_{j}^{2}$, where $\tilde{X}_{j}$ is the lifted vector field related to $X_{j}$. 
Theorem 3.1. Let $p \in(1, \infty)$. For all $u \in C_{0}^{\infty}\left(\mathbb{R}^{N}\right)$ we have

$$
\left\|\tilde{X}^{2} u\right\|_{L^{p}} \leq c\left\{\left\|\Delta_{\tilde{X}} u\right\|_{L^{p}}+\|\tilde{X} u\|_{L^{p}}+\|u\|_{L^{p}}\right\}
$$

where the constant $c$ does not depend on $u$.

Proof. In [20] (see also [1]) it has been proved that for every test function $a$ (for the existence of test functions the reader can consult e.g. [11]) there exist an operator of type two $P^{*}$ and $q$ operators of type one $S_{j}^{*}$ such that for every $u \in C_{0}^{\infty}\left(\mathbb{R}^{n}\right)$

$$
P^{*} \Delta_{\tilde{X}} u=a u+\sum_{j} S_{j}^{*} u .
$$

We apply $\tilde{X}_{h} \tilde{X}_{k}, k, j=1, \ldots, q$, to both sides of $(3)$, then

$$
\tilde{X}_{h} \tilde{X}_{k}\left(P^{*} \Delta_{\tilde{X}} u\right)=\tilde{X}_{h} \tilde{X}_{k}(a u)+\sum_{j} \tilde{X}_{h} \tilde{X}_{k}\left(S_{j}^{*} u\right) .
$$

Now, using Theorem 8 and Corollary of Theorem 9, $\S 14$ in [20] (see also Lemma 2.9 and Theorem 2.10 in [1) it follows

$$
\tilde{X}_{h} \tilde{X}_{k}\left(P^{*} \Delta_{\tilde{X}} u\right)=\tilde{X}_{h} \tilde{X}_{k}(a u)+\sum_{j} \sum_{l}\left(T_{l}^{j} \tilde{X}_{l} u+T_{0}^{j}\right)
$$

where $T_{l}^{j}$ and $T_{0}^{j}$ are suitable operators of type 0 . Denoting by $T$ the operator $T=\tilde{X}_{h} \tilde{X}_{k} P^{*}$ from (4) we get

$$
\left\|\tilde{X}_{h} \tilde{X}_{k}(a u)\right\|_{p} \leq\left\|T \Delta_{\tilde{X}} u\right\|_{p}+\sum_{j} \sum_{l}\left\|T_{l}^{j} \tilde{X}_{l} u\right\|_{p}+\left\|T_{0}^{j} u\right\|_{p} .
$$

Since all the operators are of type 0 , by Theorem 11 ( $\$ 16$ in [20]), we obtain that

$$
\left\|\tilde{X}_{h} \tilde{X}_{k}(a u)\right\|_{p} \leq c\left(\left\|\Delta_{\tilde{X}} u\right\|_{p}+\|\tilde{X} u\|_{p}+\|u\|_{p}\right),
$$

from which the theorem follows.

Remark 3.1. We point out that the statement in our previous theorem is contained in Theorem 3.2 in [1]. However we provided our proof to show that in our (simpler) case there is no dependence on the constant $c$ on the radius of the support of $u$.

Theorem 3.2. Let $p \in(1,+\infty)$, for all $u \in W_{0}^{2, p}(\Omega)$ we have

$$
\left\|X^{2} u\right\|_{L^{p}(\Omega)} \leq c\left(\left\|\Delta_{X} u\right\|_{L^{p}(\Omega)}+\|u\|_{L^{p}(\Omega)}\right) .
$$

Proof. Let $u$ be a function in $C_{0}^{\infty}(\Omega)$ and let $\eta(t)$ be a function in $C_{0}^{\infty}\left(\mathbb{R}^{N-n}\right)$, $0 \leq \eta \leq 1, \eta \equiv 1$ in $I_{r}, \eta=0$ in $I_{2 r},|\tilde{X} \eta|^{2}+\left|\tilde{X}^{2} \eta\right| \leq \frac{c}{r^{2}}$ where $I_{r}$ is a ball with radius $r$ and $I_{2 r}$ is a ball with radius $2 r$. Hence $\eta u$ is in $C_{0}^{\infty}\left(\mathbb{R}^{N}\right)$ and 
therefore

$$
\begin{gathered}
\left|I_{r}\right| \int_{B}\left|X^{2} u\right|^{p} d x \leq \int_{B \times I_{r}}\left|X^{2} u\right|^{p} d x d t \\
=\int_{B \times I_{r}}\left|\tilde{X}^{2}(\eta u)\right|^{p} d x d t \leq \int_{B \times I_{2 r}}\left|\tilde{X}^{2}(\eta u)\right|^{p} d x d t \\
\leq c\left(\int_{B \times I_{2 r}}\left|\Delta_{\tilde{X}}(\eta u)\right|^{p} d x d t+\int_{B \times I_{2 r}}|\tilde{X}(\eta u)|^{p} d x d t+\int_{B \times I_{2 r}}|\eta u|^{p} d x d t\right) \\
\leq c\left(\int_{B \times I_{2 r}}\left|\Delta_{X} u\right|^{p} d x d t+\int_{B \times I_{2 r}}|X u|^{p} d x d t+\int_{B \times I_{2 r}}|u|^{p} d x d t\right) \\
\leq c\left|I_{2 r}\right|\left(\int_{B}\left|\Delta_{X} u\right|^{p} d x+\int_{B}|X u|^{p} d x+\int_{B}|u|^{p} d x\right) .
\end{gathered}
$$

This implies that

$$
\left\|X^{2} u\right\|_{L^{p}} \leq c\left\{\left\|\Delta_{X} u\right\|_{L^{p}}+\|X u\|_{L^{p}}+\|u\|_{L^{p}}\right\}
$$

for all $u \in C_{0}^{\infty}(\Omega)$. By interpolation and density arguments we obtain

$$
\left\|X^{2} u\right\|_{L^{p}} \leq c\left(\left\|\Delta_{X} u\right\|_{L^{p}}+\|u\|_{L^{p}}\right) \quad \text { for all } u \in W_{0}^{2, p}(\Omega) .
$$

Now, we can get rid of the last term in (5) arguing as the Euclidean case (see [12], Lemma 9.17).

Theorem 3.3. Let $p \in(1, \infty)$. Then there exists a constant $\mathcal{C}_{p}$ such that for all $u \in W_{0}^{2, p}(\Omega)$ we have

$$
\left\|X^{2} u\right\|_{L^{p}(\Omega)} \leq \mathcal{C}_{p}\left\|\Delta_{X} u\right\|_{L^{p}(\Omega)} .
$$

Proof. If [6] is not true, then for all $m \in \mathbb{N}$ there exists $v_{m} \in W_{0}^{2, p}(\Omega)$ (that we can suppose with $L^{p}$ norm equal to 1 ) such that

$$
\left\|X^{2} v_{m}\right\|_{L^{p}(\Omega)}>m\left\|\Delta_{X} v_{m}\right\|_{L^{p}(\Omega)} .
$$

Since (5) holds, we have that $\left\|\Delta_{X} v_{m}\right\|_{L^{p}} \rightarrow 0$. Then, by (5) the sequence $v_{m}$ is bounded in $W_{0}^{2, p}(\Omega)$. By the weak compactness of bounded sets in $W^{2, p}(\Omega)$, there exists a subsequence $\left\{v_{m}\right\}$ converging weakly to a function $v \in W_{0}^{2, p}(\Omega)$ satisfying $\|v\|_{L^{p}}=1$. From the weak convergence and the convergence to zero in $L^{p}(\Omega)$ of $\Delta_{X} v_{m}$, we must have

$$
\int_{\Omega} g \Delta_{X} v=0, \quad \forall g \in L^{\frac{p}{p-1}}(\Omega) .
$$

Hence $\Delta_{X} v=0$ and from the representation formula $v=0$ which contradicts $\|v\|_{L^{p}}=1$.

Remark 3.2. Let us consider the Heisenberg group $\mathbb{H}$ which is $\mathbb{R}^{3}$ endowed with the group multiplication

$$
\left(\mu_{1}, \nu_{1}, \tau_{1}\right) \cdot\left(\mu_{2}, \nu_{2}, \tau_{2}\right)=\left(\mu_{1}+\mu_{2}, \nu_{1}+\nu_{2}, \tau_{1}+\tau_{2}+\frac{1}{2}\left(\nu_{1} \mu_{2}-\mu_{1} \nu_{2}\right)\right) .
$$

The canonical horizontal vector fields are

$$
\hat{X}_{1}=\frac{\partial}{\partial \mu}-\frac{\nu}{2} \frac{\partial}{\partial \tau}
$$




$$
\hat{X}_{2}=\frac{\partial}{\partial \nu}+\frac{\mu}{2} \frac{\partial}{\partial \tau} \text {. }
$$

In this case the spectral theory of the sub-Laplacian $\Delta_{\hat{X}}$ (see [6, 22, 23]) gives

$$
\iiint_{\mathbb{R}^{3}}\left(\frac{\partial w}{\partial \tau}\right)^{2} d \mu d \nu d \tau \leq \iiint_{\mathbb{R}^{3}}\left(\Delta_{\hat{X}} w\right)^{2} d \mu d \nu d \tau, \text { for all } w \in C_{0}^{\infty}\left(\mathbb{R}^{3}\right)
$$

which leads to $\mathcal{C}_{2}=\sqrt{3}$ in the Heisenberg group.

The lifting method adds an extra variable to the Grušin vector fields (see Section $\S 5$ below) leads to the the polarized Heisenberg group $\mathbb{H}^{\text {pol }}$, which is $\mathbb{R}^{3}$ endowed with the group multiplication

$$
\left(x_{1}, y_{1}, t_{1}\right) \cdot\left(x_{2}, y_{2}, t_{2}\right)=\left(x_{1}+x_{2}, y_{1}+y_{2}, t_{1}+t_{2}+x_{1} y_{2}\right) .
$$

In this case the canonical horizontal vector fields are

$$
\begin{gathered}
\tilde{X}_{1}=\frac{\partial}{\partial x} \\
\tilde{X}_{2}=\frac{\partial}{\partial y}+x \frac{\partial}{\partial t} .
\end{gathered}
$$

The two groups are isomorphic and the isomorphism $\Phi: \mathbb{H} \rightarrow \mathbb{H}^{\text {pol }}$ is given by

$$
\Phi(\mu, \nu, \tau)=\left(\mu, \nu, \tau+\frac{1}{2} \mu \nu\right)
$$

This induces a change of variables

$$
\left\{\begin{array}{l}
x=\mu \\
y=\nu \\
t=\tau+\frac{1}{2} \mu \nu
\end{array}\right.
$$

that has its Jacobian equal to 1 and transforms $\hat{X}_{i}$ to $\tilde{X}_{i}$ and. This shows that we have

$$
\iiint_{\mathbb{R}^{3}}\left(\frac{\partial w}{\partial t}\right)^{2} d x d y d t \leq \iiint_{\mathbb{R}^{3}}\left(\Delta_{\tilde{X}} w\right)^{2} d x d y d t, \text { for all } w \in C_{0}^{\infty}\left(\mathbb{R}^{3}\right)
$$

which leads to $\mathcal{C}_{2}=\sqrt{3}$ in the polarized Heisenberg group.

We consider now $u(x, t) \in C_{0}^{\infty}\left(\mathbb{R}^{2}\right)$ and for each $n \in \mathbb{N}$ the function $v(y) \in C_{0}^{\infty}(\mathbb{R})$ defined by $v_{n} \equiv 1$ on $[-n, n], v_{n} \equiv 0$ on $(-\infty,-(n+1)]$ and on $[n+1,+\infty)$, while for each $n$ on the intervals $(-(n+1),-n)$ and $(n, n+1)$ we require similar behavior. In this way there exist three constants $c_{1}, c_{2}, c_{3}$ such that

$$
\int_{\mathbb{R}} v_{n}^{2}(y) d y=2 n+c_{1}, \int_{\mathbb{R}}\left(v_{n}^{\prime}(y)\right)^{2} d y=c_{2}, \int_{\mathbb{R}}\left(v_{n}^{\prime \prime}(y)\right)^{2} d y=c_{3} .
$$

Using inequality (8) for the function $w(x, y, t)=u(x, t) v_{n}(y)$ gives

$$
\begin{aligned}
\iiint_{\mathbb{R}^{3}}\left(\frac{\partial u}{\partial t}\right)^{2} & v_{n}^{2} d x d y d t \\
& \leq \iiint_{\mathbb{R}^{3}}\left(\left(\frac{\partial^{2} u}{\partial x^{2}}+x^{2} \frac{\partial^{2} u}{\partial t^{2}}\right) v_{n}+u v_{n}^{\prime \prime}+2 x \frac{\partial u}{\partial t} v_{n}^{\prime}\right)^{2} d x d y d t
\end{aligned}
$$


Dividing this last inequality by $\int_{\mathbb{R}} v_{n}^{2}(y) d y$, using the facts that $v_{n} v_{n}^{\prime}=$ $\frac{1}{2}\left(v_{n}^{2}\right)^{\prime}, v_{n}^{\prime} v_{n}^{\prime \prime}=\frac{1}{2}\left(\left(v_{n}^{\prime}\right)^{2}\right)^{\prime}, v_{n} v_{n}^{\prime \prime}=\left(v_{n} v_{n}^{\prime}\right)^{\prime}-\left(v_{n}^{\prime}\right)^{2}$ and then letting $n \rightarrow \infty$ gives inequality $(2)$. Integration by parts similar to that of [6] leads to the following result:

Theorem 3.4. For the Grušin vector fields $X=\left(X_{1}, X_{2}\right)$ we have

$$
\left\|X^{2} u\right\|_{L^{2}(\Omega)} \leq \sqrt{3}\left\|\Delta_{X} u\right\|_{L^{2}(\Omega)}
$$

for all $u \in W_{0}^{2,2}(\Omega)$.

\section{Subelliptic Linear OPERATORS IN NON-DIVERGENCE FORM With MEASURABLE COEFFICIENTS}

Under the assumptions listed at the beginning of Section $\S 3$, let us consider the operator

$$
\mathcal{A} u=\sum_{i, j}^{q} a_{i j}(x) X_{i} X_{j} u
$$

where $a_{i j} \in L^{\infty}(\Omega)$. We assume that $\mathcal{A}$ satisfies the Cordes condition $K_{\epsilon, \sigma}$, which means that there exists $\epsilon \in(0,1]$ and $\sigma>0$ such that for a.e. $x \in \Omega$

$$
0<\frac{1}{\sigma} \leq \sum_{i, j=1}^{q} a_{i j}^{2}(x) \leq \frac{1}{q-1+\epsilon}\left(\sum_{i=1}^{q} a_{i i}\right)^{2} .
$$

We denote by $I$ the $q \times q$ identity matrix and let $A(x)=\left\{a_{i j}(x)\right\}$.

Theorem 4.1. Let $0<\epsilon \leq 1, \sigma>0$ such that $\gamma=\sqrt{1-\epsilon} \mathcal{C}<1\left(\mathcal{C}=\mathcal{C}_{2}\right.$ is the constant of Theorem 3.3) and $\mathcal{A}$ satisfies the condition $K_{\epsilon, \sigma}$. Then for all $u \in W_{0}^{2,2}(\Omega)$ we have

$$
\left\|X^{2} u\right\|_{L^{2}(\Omega)} \leq \frac{\mathcal{C}}{1-\gamma}\|\alpha\|_{L^{\infty}(\Omega)}\|\mathcal{A} u\|_{L^{2}(\Omega)},
$$

where $\alpha(x)=\frac{\langle A(x), I\rangle}{\|A(x)\|^{2}}$.

Proof. Since $\mathcal{A}$ satisfies the condition $K_{\epsilon, \sigma}$ then $A$ satisfies (see [6], Theorem 2.1)

$$
\left|\sum_{i=1}^{q} m_{i i}-\alpha(x) \sum_{i, j=1}^{q} a_{i j}(x) m_{i j}\right| \leq \sqrt{1-\epsilon}\left(\sum_{i, j=1}^{q} m_{i j}^{2}\right)^{1 / 2}
$$

for all $M \in \mathcal{M}_{q}(\mathbb{R})$. It follows that

$$
\begin{gathered}
\int_{\Omega}\left|\Delta_{X} u(x)-\alpha(x) \mathcal{A} u(x)\right|^{2} d x \leq(1-\epsilon) \int_{\Omega} \sum_{i, j=1}^{q}\left(X_{i} X_{j} u(x)\right)^{2} d x \leq \\
\leq(1-\epsilon) \mathcal{C}^{2} \int_{\Omega}\left|\Delta_{X} u(x)\right|^{2} d x .
\end{gathered}
$$

Then

$$
\left\|\Delta_{X} u-\alpha \mathcal{A} u\right\|_{L^{2}(\Omega)} \leq \gamma\left\|\Delta_{X} u\right\|_{L^{2}(\Omega)},
$$

and, since $\gamma<1$, we obtain

$$
\left\|X^{2} u\right\|_{L^{2}(\Omega)} \leq \frac{\mathcal{C}}{1-\gamma}\|\alpha\|_{\infty}\|\mathcal{A} u\|_{L^{2}(\Omega)} .
$$


As a corollary we have the following result in the Grušin plane:

Corollary 4.1. Let $0<\epsilon \leq 1$ and $\sigma>0$ such that $\gamma=\sqrt{3(1-\epsilon)}<1$ and $\mathcal{A}$ satisfies the Cordes condition $K_{\epsilon, \sigma}$. Then for all $u \in W_{0}^{2,2}(\Omega)$ we have

$$
\left\|X^{2} u\right\|_{L^{2}(\Omega)} \leq \frac{\sqrt{3}}{1-\gamma}\|\alpha\|_{L^{\infty}(\Omega)}\|\mathcal{A} u\|_{L^{2}(\Omega)},
$$

where $\alpha(x)=\frac{\langle A(x), I\rangle}{\|A(x)\|^{2}}$.

\section{5. $W_{\text {loc }}^{2,2}(\Omega)$ REGULARITY FOR QUASILINEAR EQUATIONS IN THE GRUŠIN}

PLANE

In this section we consider a non-degenerate quasilinear equation and obtain $W_{\text {loc }}^{2,2}$ estimates for its weak solutions. Let $X_{1}=\frac{\partial}{\partial x}, X_{2}=x \frac{\partial}{\partial t}$, and denote their commutator by $T=\left[X_{1}, X_{2}\right]=\frac{\partial}{\partial t}$.

Consider the non-degenerate p-laplacian equation

$$
-\sum_{i=1}^{2} X_{i}\left(\left(\lambda+|X u|^{2}\right)^{\frac{p-2}{2}} X_{i} u\right)=0, \text { in } \Omega \subset \mathbb{R}^{2}
$$

with $\lambda>0$.

A weak solution of equation (9) is a function $u$ belonging to the horizontal Sobolev space $W_{\text {loc }}^{1, p}(\Omega)$ and satisfying

$$
\sum_{i=1}^{2} \int_{\Omega}\left(\lambda+|X u|^{2}\right)^{\frac{p-2}{2}} X_{i} u X_{i} \varphi d x d t=0
$$

for all $\varphi \in W_{0}^{1, p}(\Omega)$ with compact support.

The lifting process to the Lie algebra of the polarized Heisenberg group (see [23] and Remark 3.1 in this paper) is implemented by setting

$$
\tilde{X}_{1}=\frac{\partial}{\partial x}, \quad \tilde{X}_{2}=x \frac{\partial}{\partial t}+\frac{\partial}{\partial y} .
$$

Denoting $\tilde{u}(x, y, t)=u(x, t)$ it is simple to prove that $\tilde{u}$ is a weak solution of the lifted subelliptic p-Laplacian:

$$
-\sum_{i=1}^{2} \tilde{X}_{i}\left(\left(\lambda+|\tilde{X} u|^{2}\right)^{\frac{p-2}{2}} \tilde{X}_{i} u\right)=0, \text { in } \tilde{\Omega}=\Omega \times(-a, a) .
$$

Therefore we can use some of the methods and results valid in the Heisenberg group to obtain regularity properties for $\tilde{u}$ and then get similar conclusions for $u$. Observe that by Remark 3.2 and Theorem 3.4 the results from [5, 6] regarding the second order differentiability of the $p$-harmonic functions obtained in the regular Heisenberg group are valid in the polarized Heisenberg group too. Therefore the following theorems hold in the Grušin plane. 
Theorem 5.1. [5] Let $1<p<4, u_{\lambda} \in W_{\text {loc }}^{1, p}(\Omega)$ be a weak solution of (9), $\left(x_{0}, t_{0}\right) \in \Omega$ and $r>0$ such that $B\left(\left(x_{0}, t_{0}\right), 3 r\right) \subset \Omega$. Then there exists a number $k \in \mathbb{N}$ (depending only on $p$ ) such that

$$
\int_{B\left(\left(x_{0}, t_{0}\right), r / 2^{k+1}\right)}\left|T u_{\lambda}\right|^{p} d x d t \leq c \int_{B\left(\left(x_{0}, t_{0}\right), 2 r\right)}\left(\left(\lambda+\left|X u_{\lambda}\right|^{2}\right)^{\frac{p}{2}}+\left|u_{\lambda}\right|^{p}\right) d x d t,
$$

which implies that $T u_{\lambda} \in L_{l o c}^{p}(\Omega)$.

Theorem 5.2. [5] Let $2 \leq p<4$ and $u_{\lambda} \in W_{\text {loc }}^{1, p}(\Omega)$ be a weak solution of (9). Then, if $B\left(\left(x_{0}, t_{0}\right), 3 r\right) \subset \Omega$, there exists $k \in \mathbb{N}$ depending only on $p$ such that

$$
\begin{aligned}
& \int_{B\left(\left(x_{0}, t_{0}\right), r / 2^{k+2}\right)}\left(\lambda+\left|X u_{\lambda}\right|^{2}\right)^{\frac{p-2}{2}}\left|X^{2} u_{\lambda}\right|^{2} d x d t \\
& \leq c \int_{B\left(\left(x_{0}, t_{0}\right), 2 r\right)}\left(\left(\lambda+\left|X u_{\lambda}\right|^{2}\right)^{\frac{p}{2}}+\left|u_{\lambda}\right|^{p}\right) d x d t,
\end{aligned}
$$

from which it follows that $u_{\lambda} \in W_{l o c}^{2,2}(\Omega)$.

Theorem 5.3. 5] Let $\frac{\sqrt{17}-1}{2} \leq p \leq 2$ and $u_{\lambda} \in W_{\text {loc }}^{1, p}(\Omega)$ be a weak solution of (9). If $B\left(\left(x_{0}, t_{0}\right), 3 r\right) \subset \Omega$ then there exists $k \in \mathbb{N}$ depending only on $p$ such that

$$
\begin{aligned}
& \int_{B\left(\left(x_{0}, t_{0}\right), r / 2^{k+3}\right)}\left|X^{2} u_{\lambda}\right|^{p} d x d t \\
\leq & c\left\{\lambda^{\frac{p-2}{2}}\|u\|_{C^{\delta}\left(B\left(\left(x_{0}, t_{0}\right), r / 2^{k+1}\right)\right.}^{2-p} \int_{B\left(\left(x_{0}, t_{0}\right), 2 r\right)}\left(\left(\lambda+\left|X u_{\lambda}\right|^{2}\right)^{\frac{p}{2}}+\left|u_{\lambda}\right|^{p}\right) d x d t\right. \\
+ & \left.\lambda^{\frac{p-2}{2}}\|u\|_{L^{2}\left(B\left(\left(x_{0}, t_{0}\right), r / 2^{k+1}\right)\right)}^{2}+\int_{B\left(\left(x_{0}, t_{0}\right), 2 r\right)}\left(\left(\lambda+\left|X u_{\lambda}\right|^{2}\right)^{\frac{p}{2}}+\left|u_{\lambda}\right|^{p}\right) d x d t\right\},
\end{aligned}
$$

and hence $u_{\lambda} \in W_{\text {loc }}^{2, p}(\Omega)$.

Theorem 5.4. [6]

(1) For $\frac{\sqrt{17}-1}{2} \leq p<4$ any weak solution of the nondegenerate subelliptic $p$-Laplacian equation (6) belongs to $W_{\mathrm{loc}}^{2,2}(\Omega)$.

(2) For $\frac{\sqrt{17}-1}{2} \leq p<\frac{5+\sqrt{5}}{2}$ any weak solution of the subelliptic $p$-Laplacian equation (1) belongs to $H W_{\mathrm{loc}}^{2,2}(\Omega)$.

Remark 5.1. Observe that the intervals given by Theorem 5.4 contain $p=3$, which is the homogeneous dimension near the singular line $x=0$.

\section{6. $C^{1, \alpha}$ estimates in the Grušin Plane}

In this section we prove $C^{1, \alpha}$ regularity for the p-harmonic functions in the Grušin plane. We list the following theorems that are useful in the proof of our main result. 
Theorem 6.1. Let $u \in W_{\text {loc }}^{2, p}(\Omega),\left(x_{0}, t_{0}\right) \in \Omega$ and $r>0$ such that $B\left(\left(x_{0}, t_{0}\right), r\right) \subset$ $\Omega$. Then for all $\delta>0$ there exists a constant $c(\delta)$ such that

$$
\|X u\|_{L^{p}\left(B\left(\left(x_{0}, t_{0}\right), r\right)\right)} \leq \delta\left\|X^{2} u\right\|_{L^{p}\left(B\left(\left(x_{0}, t_{0}\right), r\right)\right)}+c(\delta)\|u\|_{L^{p}\left(B\left(\left(x_{0}, t_{0}\right), r\right)\right)} .
$$

Proof. See Lemma 4 in [24].

Consider the operator

$$
\mathcal{A} u=\sum_{i, j}^{2} a_{i j}(x) X_{i} X_{j} u
$$

where $a_{i j} \in L^{\infty}(\Omega)$.

Theorem 6.2. Fix $1<p<\infty$ and let $0<\epsilon \leq 1$, such that $\epsilon \mathcal{C}_{p}<1$. Suppose that

$$
\left|\Delta_{X} u(x, t)-\mathcal{A} u(x, t)\right| \leq \epsilon\left|X^{2} u(x, t)\right|,
$$

for a. e. $(x, t) \in \Omega$ and for all $u \in W_{0}^{2, p}(\Omega)$. Then $\mathcal{A}: W_{0}^{2, p}(\Omega) \rightarrow L^{p}(\Omega)$ is an isomorphism and there exists $c>0$ such that

$$
\left\|X^{2} u\right\|_{L^{p}(\Omega)} \leq c\|\mathcal{A} u\|_{L^{p}(\Omega)}
$$

for all $u \in W_{0}^{2, p}(\Omega)$.

Proof. Since $\left\|\Delta_{X} u-\mathcal{A} u\right\|_{L^{p}(\Omega)} \leq \epsilon \mathcal{C}\left\|\Delta_{X} u\right\|_{L^{p}(\Omega)}$ then $\mathcal{A}$ is near to $\Delta_{X}$ from which it follows that $\mathcal{A}$ is an isomorphism and that (13) is true (see [2]).

Consider now the case when $\lambda=\frac{1}{m}$. Then the corresponding nondegenerate $p$-Laplace equation is

$$
\sum_{i=1}^{2} X_{i}\left(\left(\frac{1}{m}+|X u|^{2}\right)^{\frac{p-2}{2}} X_{i} u\right)=0 .
$$

Theorems 5.2 and 5.3 show that for the range $\frac{\sqrt{17}-1}{2} \leq p<4$ weak solutions $u_{m}$ satisfy also the differentiated version of equation (14)

$$
L_{m}\left(u_{m}\right)=\sum_{i=1}^{2} a_{i j}^{m} X_{i} X_{j} u_{m}=0,
$$

where

$$
a_{i j}^{m}=\delta_{i j}+(p-2) \frac{X_{i} u_{m} X_{j} u_{m}}{1 / m+\left|X u_{m}\right|^{2}} .
$$

The homogeneous dimension of a compact $E \subset \subset \Omega$ is 2 if $E$ does not intersect the line $x=0$, it is 3 if $E$ intersects the line $x=0$. In any case we denote by $Q$ the homogeneous dimension. For a fixed, but arbitrary small positive constant $\gamma$ denote

$$
\tilde{\mathcal{C}}=\sup \left\{\mathcal{C}_{p}, p \in\left[\frac{\sqrt{17}-1}{2}, Q+\gamma\right]\right\}
$$

We remark that we have

$$
\left|L_{m} v(x)-\Delta_{X} v(x)\right| \leq|p-2|\left|X^{2} v(x)\right|
$$


for a.e. $x \in \Omega$ and for all $v \in H W_{0}^{2, s}(\Omega)$, so the operator $L_{m}$ satisfies the condition 12 with $\epsilon \leq \min \left\{\frac{1}{\tilde{\mathcal{C}}},|p-2|\right\}$.

Theorems 6.1 and 6.2 imply the following result.

Theorem 6.3. Let us suppose that the assumptions of Theorem 6.2 hold. Let $\left(x_{0}, t_{0}\right) \in \Omega$ and $r>0$ such that $B\left(\left(x_{0}, t_{0}\right), 3 r\right) \subset \Omega$. Then for all $u \in W_{\mathrm{loc}}^{2, p}(\Omega)$ we have

$$
\left\|X^{2} u\right\|_{L^{p}\left(B\left(\left(x_{0}, t_{0}\right), r\right)\right)} \leq c\left(\|\mathcal{A} u\|_{L^{p}\left(B\left(\left(x_{0}, t_{0}\right), 2 r\right)\right)}+\|u\|_{L^{p}\left(B\left(\left(x_{0}, t_{0}\right), 2 r\right)\right)}\right) .
$$

We are now able to prove our main result.

Theorem 6.4. Consider

$$
\max \left\{\frac{\sqrt{17}-1}{2}, 2-\frac{1}{\tilde{\mathcal{C}}}\right\} \leq p \leq 2+\frac{1}{\tilde{\mathcal{C}}} .
$$

If $u \in W^{1, p}(\Omega)$ is a p-harmonic function, then there exists $0<\alpha<1$ such that $u \in C_{\mathrm{loc}}^{1, \alpha}(\Omega)$.

Proof. Consider $\left(x_{0}, t_{0}\right) \in \Omega$ and $r>0$ such that $B\left(\left(x_{0}, t_{0}\right), 4 r\right) \subset \Omega$. Without loss of generality we can suppose that $X u_{m} \in W^{1, p}\left(B\left(\left(x_{0}, t_{0}\right), 3 r\right) \subset \Omega\right)$ with uniform bounds in $m$. If $Q=2$ and we choose $1<\beta<2$ close enough to 1 such that

$$
\frac{2 \beta}{2-\beta} \leq 2+\gamma
$$

then we have

$$
W_{0}^{1, \beta}\left(\Omega^{\prime}\right) \hookrightarrow L^{\frac{2 \beta}{2-\beta}}\left(\Omega^{\prime}\right)
$$

for any $\Omega^{\prime} \subset \subset \Omega$. Consider a cut-off function $\eta$ between the balls $\left.B\left(\left(x_{0}, t_{0}\right), r\right)\right)$ and $\left.B\left(\left(x_{0}, t_{0}\right), 2 r\right)\right)$. Then for $q_{0}=\frac{2 \beta}{2-\beta}$ we have

$\left\|L_{m}\left(\eta^{2} u_{m}\right)\right\|_{\left.L^{q_{0}}\left(B\left(\left(x_{0}, t_{0}\right), 2 r\right)\right)\right)} \leq c\left(\left\|u_{m}\right\|_{L^{q_{0}(\operatorname{supp} \eta)}}+\left\|X u_{m}\right\|_{L^{q_{0}}(\operatorname{supp} \eta)}\right)<+\infty$.

By Theorem 6.3 and the fact that the $W^{1, p}$ norm of $u_{m}$ is uniformly bounded on supp $\eta$ we get that $\left.\eta u_{m} \in W^{2, q_{0}}\left(B\left(\left(x_{0}, t_{0}\right), 2 r\right)\right)\right)$ with uniform bounds. Since $\left.\left.W_{0}^{1, q_{0}}\left(B\left(\left(x_{0}, t_{0}\right), 2 r\right)\right)\right) \hookrightarrow C^{\frac{2 \beta-2}{\beta}}\left(B\left(\left(x_{0}, t_{0}\right), 2 r\right)\right)\right)$ we have that

$$
\left.u_{m} \in C^{1, \alpha}\left(B\left(\left(x_{0}, t_{0}\right), 2 r\right)\right)\right) \text { with } \quad \alpha=\frac{2 \beta-2}{\beta} .
$$

Since the estimates for $u_{m}$ are uniform in $m$, we conclude that $u \in C_{\text {loc }}^{1, \alpha}(\Omega)$.

A similar proof can be applied in the case $Q=3$. We use the embedding

$$
W_{0}^{1,2}\left(\Omega^{\prime}\right) \hookrightarrow L^{6}\left(\Omega^{\prime}\right)
$$

to get $u \in W_{\mathrm{loc}}^{2,6}(\Omega)$ and then $u \in C_{\mathrm{loc}}^{1,1 / 2}(\Omega)$.

\section{REFERENCES}

[1] M. Bramanti, L. Brandolini, $L^{p}$ estimates for nonvariational hypoelliptic operators with VMO coefficients, Trans. Amer. Math. Soc., 352 n.2 (2000) 781-822.

[2] S. Campanato, On the condition of nearness between operators Ann. Mat. Pura Appl. CLXVII (1994) 243-256.

[3] L. Capogna, Regularity of quasilinear equations in the Heisenberg group, Comm. Pure Appl. Math. 50 (1997) 867-889. 
[4] W. L. Chow, Über systeme von linearen partiellen differentialgleichungen ersten ordnug, Math. Ann. 117 (1939), 98-105.

[5] A. Domokos, Differentiability of solutions for the non-degenerate p-Laplacian in the Heisenberg group, J. Differential Equations 204 (2004), 439-470.

[6] A. Domokos, J. J. Manfredi, Subelliptic Cordes estimate, Proc. A.M.S. 133 (2005), $1047-1056$

[7] A. Domokos, J. J. Manfredi, $C^{1, \alpha}$-regularity for p-harmonic functions in the Heisenberg group for $p$ near 2, Contemporary Mathematics, 370 (2005), 17 - 23.

[8] C.L. Fefferman, A. Sanchez-Calle, Fundamental solutions for second order subelliptic operators Ann. of Math. 124 (1986), 247-272.

[9] G. B. Folland, Subelliptic estimates and function spaces on nilpotent Lie groups, Arkiv för Math. 13 (1975), 161-207.

[10] G. B. Folland, Applications of analysis on nilpotent groups to partial differential equations, Bulletin of AMS 83 (1977), 912-930.

[11] B. Franchi, R. Serapioni, F. Serra-Cassano Approximation and imbedding theorems associated with Lipschitz continuous vector fields Boll.U.M.I. (7)11-B (1997), 83-117.

[12] D. Gilbarg, N. S. Trudinger, Elliptic partial differential equations of second order, Springer-Verlag 1983.

[13] P.Hajłasz, P.Koskela, Sobolev met Poincare, Memoirs of the American Mathematical Society 688, (2000).

[14] L. Hörmander, Hypoelliptic second order differential equations, Acta Math. 119 (1967), 147-171.

[15] D. Jerison, The Poincaré inequality for vector fields satisfying Hormander's condition, Duke Math. J. 53 (1986), 503-523.

[16] G. Lu, Existence and size estimates for the Green's functions of differentail operators constructed from degenerate vector fields, Comm. P.D.E. 17, n. 7-8, (1992), 1213-1251.

[17] G. Lu, On Harnack's inequality for a class of strongly degenerate Schrödinger operators formed by vector fields, Diff. and Integral Equations 7, n. 1, (1994), 73-100.

[18] A. Nagel, E. Stein, S. Wainger, Balls and metrics defined by vector fields I: Basic Properties, Acta Math., 155 (1985), 130-147.

[19] A. Nagel, E. Stein, Differentiable control metrics and scaled bump functions, Jour. of Diff. Geometry, 57 (2001), 465-492.

[20] L. P. Rothschild, E. M. Stein, Hypoelliptic differential operators and nilpotent groups, Acta Math., 137 (1976), 247-320.

[21] A.Sánchez-Calle, Fundamental solutions and geometry of sum of squares of vector fields, Inv. Math. 78 (1984), 143-160.

[22] R. S. Strichartz, $L^{p}$ Harmonic analysis and Radon transforms on the Heisenberg group, J. Funct. Analysis, 96 (1991), 350-406.

[23] S. Thangavelu, Harmonic analysis on the Heisenberg group, Progress in Mathematics vol. 159, Birkhäuser, Boston, 1998.

[24] C. J. Xu, Subelliptic variational problems, Bull. Soc. Math. France 118 n.2 (1990) $147-169$.

Dipartimento di Matematica e Informatica, Università di Catania, Viale A.

Doria 6, 95125, Catania, Italy

E-mail address: difazio@dmi.unict.it

Department of Mathematics and Statistics, California State University

Sacramento, 6000 J Street, Sacramento, CA 95819, USA

E-mail address: domokos@csus.edu

Dipartimento di Matematica e Informatica, Università di Catania, Viale A.

Doria 6, 95125, Catania, Italy

E-mail address: fanciullo@dmi.unict.it

Department of Mathematics, University of Pittsburgh, 301 Thackeray Hall, 15260, Pittsburgh, PA, USA

E-mail address: manfredi@pitt.edu 